\title{
Increased Neurotransmitter Release at the Neuromuscular Junction in a Mouse Model of Polyglutamine Disease
}

\author{
José L. Rozas, ${ }^{1,3}$ Leonardo Gómez-Sánchez, ${ }^{1,3}$ Cristina Tomás-Zapico, ${ }^{2,3}$ José J. Lucas, ${ }^{2,3}$ and Rafael Fernández-Chacón ${ }^{1,3}$ \\ ${ }^{1}$ Instituto de Biomedicina de Sevilla Hospital Universitario Virgen del Rocío/Consejo Superior de Investigaciones Científicas (CSIC)/Universidad de Sevilla, \\ Departamento de Fisiología Médica y Biofísica Universidad de Sevilla, 41009 Sevilla, Spain, ${ }^{2}$ Centro de Biología Molecular Severo Ochoa, CSIC/Universidad \\ Autónoma de Madrid, 28049 Madrid, Spain, and ${ }^{3}$ Centro de Investigación Biomédica en Red de Enfermedades Neurodegenerativas (CIBERNED), Spain
}

In Huntington's disease (HD), the expansion of polyglutamine (polyQ) repeats at the $\mathrm{N}$ terminus of the ubiquitous protein huntingtin (htt) leads to neurodegeneration in specific brain areas. Neurons degenerating in HD develop synaptic dysfunctions. However, it is unknown whether mutant htt impacts synaptic function in general. To investigate that, we have focused on the nerve terminals of motor neurons that typically do not degenerate in HD. Here, we have studied synaptic transmission at the neuromuscular junction of transgenic mice expressing a mutant form of htt (R6/1 mice). We have found that the size and frequency of miniature endplate potentials are similar in R6/1 and control mice. In contrast, the amplitude of evoked endplate potentials in R6/1 mice is increased compared to controls. Consistent with a presynaptic increase of release probability, synaptic depression under high-frequency stimulation is higher in R6/1 mice. In addition, no changes were detected in the size and dynamics of the recycling synaptic vesicle pool. Moreover, we have found increased amounts of the synaptic vesicle proteins synaptobrevin 1,2/VAMP 1,2 and cysteine string protein- $\alpha$, and the SNARE protein SNAP-25, concomitant with normal levels of other synaptic vesicle markers. Our results reveal that the transgenic expression of a mutant form of htt leads to an unexpected gain of synaptic function. That phenotype is likely not secondary to neurodegeneration and might be due to a primary deregulation in synaptic protein levels. Our findings could be relevant to understand synaptic toxic effects of proteins with abnormal polyQ repeats.

\section{Introduction}

Polyglutamine (polyQ) diseases are inherited neurological disorders caused by CAG triplet-repeat expansions in several genes that confer toxicity to the mutated proteins (Orr and Zoghbi, 2007). Huntington's disease (HD), the most common polyglutamine disease, is caused by an abnormally long polyQ repeat in the N-terminal region of hungtingtin (htt) (The Huntington's Disease Collaborative Research Group, 1993). Although htt is ubiquitously expressed in neuronal and non-neuronal cells, the most vulnerable neurons in $\mathrm{HD}$ are the striatal medium spiny neurons and the cortical pyramidal neurons. Likely, mutant-htt becomes toxic through multiple molecular mechanisms, including synaptic dysfunction (Smith et al., 2005; Rozas et al., 2010). Detailed studies of synaptic alterations in HD mouse models in the brain regions that typically degenerated in HD have reported different phenotypes depending on synaptic type, brain region, and level of degeneration (Zeron et al., 2002; Cepeda et al., 2003,

\footnotetext{
Received April 20, 2010; revised Nov. 7, 2010; accepted Nov. 10, 2010.

This work has been supported by grants from the Instituto de Salud Carlos III (Centro de Investigación Biomédica en Red de Enfermedades Neurodegenerativas), the Spanish Ministry of Science and Innovation (BFU2007-66008 to R.F-C., SAF2009-08233 to J.J.L., and Juan de la Cierva contracts to J.L.R. and C.T.-Z.), Junta de Andalucía (P07-CVI02854, P06-CVI-02392), Comunidad Autónoma de Madrid, Fundación Ramón Areces, and Fondo Europeo de Desarrollo Regional. We are grateful to Dr. Joan Blasi and Dr. Thomas Südhof for generous gifts of antibodies and to Dr. Maria Luz Montesinos for advice.

Correspondence should be addressed to Rafael Fernández-Chacón, Instituto de Biomedicina de Sevilla Hospital Universitario Virgen del Rocío/CSIC/Universidad de Sevilla, Departamento de Fisiología Médica y Biofísica Universidad de Sevilla, and CIBERNED, Avenida Sánchez-Pizjuán 4, 41009 Sevilla, Spain. E-mail: rfchacon@us.es.

DOI:10.1523/JNEUROSCI.2011-10.2011

Copyright $\odot 2011$ the authors $\quad 0270-6474 / 11 / 311106-08 \$ 15.00 / 0$
}

2007; Smith et al., 2005; Cummings et al., 2009; Rozas et al., 2010). In any case, recognition of primary synaptic alterations in central synapses in HD is challenging because they are likely hidden by secondary changes in circuit plasticity and by neurodegeneration. A recent study on a novel HD model in Drosophila found enhanced neurotransmitter release leading to neurodegeneration in neuromuscular synapses expressing mutant htt (Romero et al., 2008). Here, to discern primary synaptic changes from changes due to neurodegeneration, we have used R6/1 mice that develop a neurodegenerative motor phenotype that is slower and moderate compared to the phenotype in R6/2 mice (Ribchester et al., 2004). Evoked neurotransmitter release from R6/1 mice motor neurons was not only robust and reliable, indeed, it was unexpectedly enhanced in comparison to control synapses.

\section{Materials and Methods}

Mice. Previously described R6/1 mice (Mangiarini et al., 1996) were used for experiments in accordance with the National Institutes of Health Guide for the Care and Use of Laboratory Animals and with the Committee of Animal Use for Research at the University of Seville. Male and female mice were used.

Electrophysiology. The levator auris longus (LAL) muscle was dissected as described previously (Angaut-Petit et al., 1987), stretched, and pinned in a Sylgard-coated perfusion chamber. High-resistance (20-40 M $\Omega$ ) glass microelectrodes filled with $3 \mathrm{~mm} \mathrm{KCl}$ were placed close to the nerve terminal $(10-50 \mu \mathrm{m})$ to minimize electrotonic filtering of postsynaptic potentials (Fatt and Katz, 1952). Nerve was drawn to a suction electrode for electrical stimulation using a Model 2100 Isolated Pulse Stimulator (A-M Systems). For the experiment described in Figure 4, two isolated 

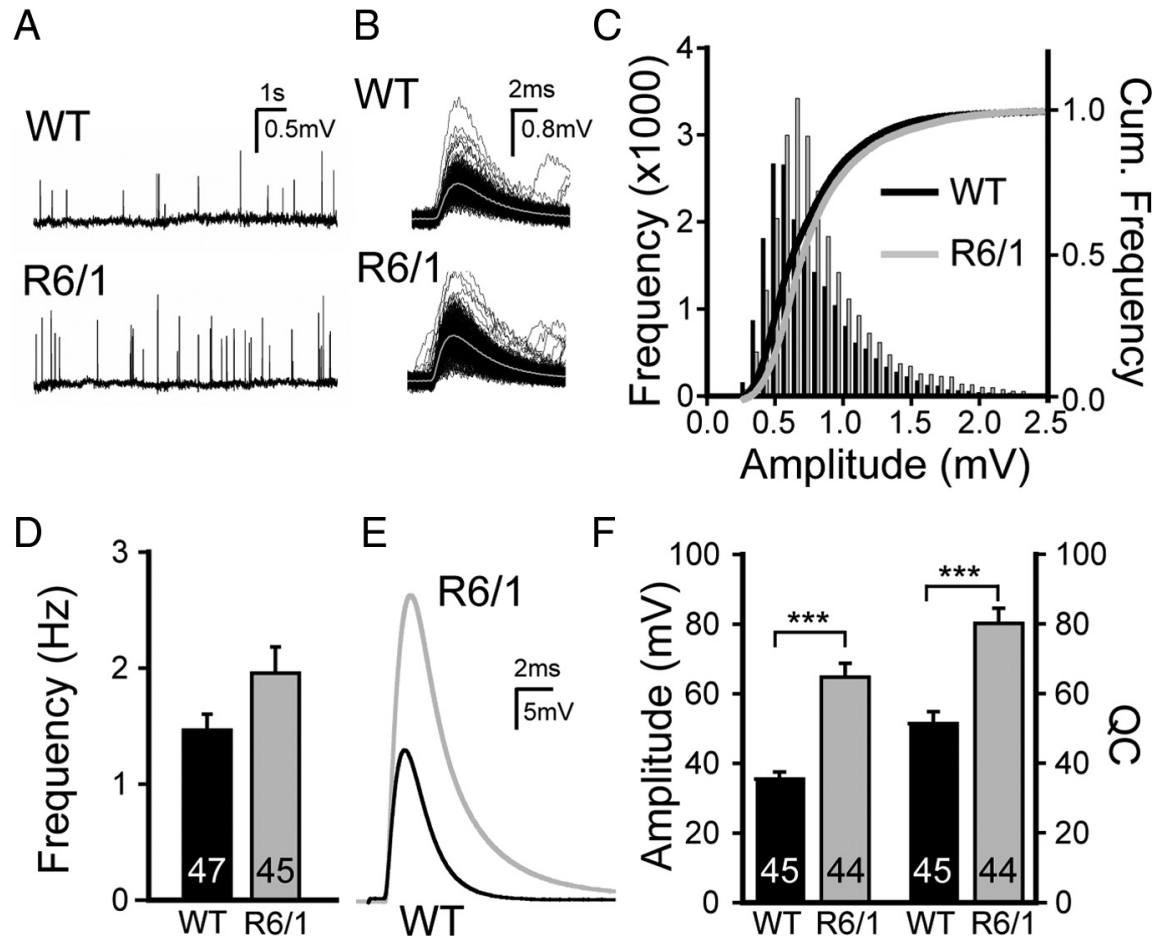

Figure 1. Increased evoked neurotransmitter release at the $\mathrm{NMJ}$ in $\mathrm{R} 6 / 1$ mice. $\boldsymbol{A}$, Exemplary recordings of spontaneous release. $\boldsymbol{B}$, Single (black) and averaged (gray) MEPPs (right) from one endplate in WT and R6/1 fibers. $\boldsymbol{C}$, Cumulative histogram of MEPP amplitude shows no size differences between WT (black) and R6/1 (gray) MEPPs. D, Spontaneous release frequency is slightly, but not significantly, increased at R6/1 synapses ( $p=0.07$, Student's $t$ test). $\boldsymbol{E}$, Exemplary recordings of EPPs from WT (black) and R6/1 (gray) mice. $\boldsymbol{F}$, Increased EPP amplitude (in millivolts) and QC released per action potential at R6/1 synapses compared to WT ${ }^{* * *} p<0.001$ for both amplitude and QC, Student's $t$ test).

pulse stimulators were used. Muscle action potentials were blocked by 1.5-2 $\mu \mathrm{M} \mu$-conotoxin (Alomone Labs). The perfusing Ringer solution contains the following (in $\mathrm{mm}$ ): $130 \mathrm{NaCl}, 5 \mathrm{KCl}, 1 \mathrm{MgSO}_{4}, 0.5$ $\mathrm{Na}_{2} \mathrm{HPO}_{4}, 20 \mathrm{NaHCO}_{3}, 2 \mathrm{CaCl}_{2}$, and 15 glucose; $\mathrm{pH}$ was 7.4. The solution was continuously bubbled with $95 \% \mathrm{O}_{2} / 5 \% \mathrm{CO}_{2}$. All experiments were performed at room temperature. Postsynaptic potentials were recorded with a EPC10 patch-clamp amplifier (HEKA) in current-clamp mode, filtered (Bessel, $2.9 \mathrm{kHz}$ ), and acquired at sampling rate of $10 \mathrm{kHz}$ using Pulse software (HEKA). Off-line analysis was performed with Axograph $\mathrm{X}$ for Windows. The amplitudes of endplate potentials (EPPs) and miniature EPPs (MEPPs) were normalized to $-75 \mathrm{mV}$, assuming $0 \mathrm{mV}$ as the reversal potential for ACh-induced current (Magleby and Stevens, $1972 \mathrm{a}, \mathrm{b})$ using the formula $\mathrm{EPP}_{\text {normalized }}=\mathrm{EPP} \times\left(-75 / V_{\mathrm{m}}\right)$, where $V_{\mathrm{m}}$ is the measured resting membrane potential. Next, the amplitude of $\mathrm{EPP}_{\text {normalized }}$ was corrected for nonlinear summation (McLachlan and Martin, 1981) using the following formula: $\mathrm{EPP}^{\prime}=\mathrm{EPP}_{\text {normalized }} /[1-$ $\left.f\left(\mathrm{EPP}_{\text {normalized }} / E\right)\right] . E$ is the difference between the membrane potential $\left(V_{\mathrm{m}}\right)$ and the equilibrium potential for ACh current $(0 \mathrm{mV})$. The value $f$ (set to 0.8 ) is a factor that improves the accuracy of the nonlinear correction by taking into account the effect of the membrane capacitance of the muscle fiber (Martin, 1976; McLachlan and Martin, 1981). The quantal content (QC; i.e., the number of ACh quanta released upon a single nerve impulse) at each neuromuscular junction (NMJ) was calculated by dividing the $\mathrm{EPP}^{\prime}$ by the normalized MEPP amplitude. To calculate QC during a stimulation train, the amplitude of MEPPs immediately recorded after the train was used.

Imaging. For FM2-10 destaining experiments, LAL muscles were dissected and stretched in Ringer solution. D-Tubocurarine was used to avoid muscle contraction, and the muscle was incubated in FM2-10 (Invitrogen, $40 \mu \mathrm{M}$ ) for $5 \mathrm{~min}$ before the stimulation was applied ( $90 \mathrm{~s}$ at 10 $\mathrm{Hz}$ ). After the stimulus, the muscle remained in FM2-10 for 5 min to complete the endocytosis and then was washed extensively. Experiments were performed at room temperature. Preparations were visualized with a Nikon Eclipse E600FN upright microscope, using $10 \times$ air and $60 \times(1.0$
NA) water-immersion objectives. Fluorescence was excited using a Polychrome IV monochromator (Till Photonics) (488 nm, 100 ms exposure time). Images were acquired at 1 $\mathrm{Hz}$ with $2 \times 2$ binning by an Andor Ixon 885 (Andor Technologies) camera using Till Vision Software. Analysis and measurements were performed in the original images using ImageJ. Images were background subtracted (from ROIs drawn beside the NMJs) and corrected for photobleaching.

Immunohistochemistry. Muscles were fixed in PBS with 4\% paraformaldehyde for $3 \mathrm{~h}$ and permeabilized in PBS Triton X-100 1\% with fetal bovine serum at 5\% (overnight incubation). After being rinsed in PBS, muscles were incubated in a solution (PBS, Triton X-100 $0.2 \%$, and FBS $10 \%$ ) containing primary antibodies for at least $8 \mathrm{~h}$ or overnight. The following primary antibodies and dilutions were used: rabbit anti-Syb1 (104 002, Synaptic Systems), 1:500; goat anti-Htt (N-18, Santa Cruz Biotechnology), 1:200; mouse anti-SV2 (developed by Dr. Buckley and obtained from the Developmental Studies Hybridoma Bank, developed under the auspices of the National Institute of Child Health and Human Development and maintained by The University of Iowa), 1:500; rabbit anti-synaptophysin 1 (101 002, Synaptic Systems), 1:300; rabbit antiSNAP-25 (I733, gift from Dr. Thomas Südhof, Stanford University, Palo Alto, CA) (McMahon and Südhof, 1995), 1:500; rabbit anti-cysteine string protein- $\alpha$ (CSP- $\alpha)$ [R807, gift from Dr. Thomas Südhof (Fernández-Chacón et al., 2004)], 1:500; rabbit anti-synaptotagmin 1,2 [V216, gift from Dr. Thomas Südhof (Ullrich and Südhof, 1994)], 1:200; and rabbit anti-Syntaxin $1 \mathrm{~A}$ and anti-Syntaxin 1B, gift from Dr. Joan Blasi (University of Barcelona, Barcelona, Spain) (Ruiz-Montasell et al., 1996), 1:50.

Secondary antibodies were used at 1:1000 concentration for $3 \mathrm{~h}$. Then, muscles rinsed in PBS were mounted using SlowFade Gold antifade (Invitrogen). Preparations were visualized using a $60 \times 1.42$ NA oilimmersion objective mounted on an Olympus Fluoview confocal microscope. For quantification of fluorescence intensity, images from WT and R6/1 junctions were collected using the same conditions of excitation, resolution, zoom, and step size ( $1 \mu \mathrm{m}$ between each image). Using ImageJ, each slice from the $z$-stack was summed to each other to obtain a unique image, and the reference marker (SV2) was used to create a mask around the NMJs. The average pixel intensity obtained from each synapse for the desired marker was divided by the SV2 average intensity to yield a normalized value. Others image treatments or filtering were avoided.

Immunoblots. For immunoblotting, LAL muscles of WT mice and R6/1 mice were extracted in Ringer solution (see above) and then homogenized in lysis solution [20 mM Tris-HCl, pH 7.4, 1\% IGEPAL, 150 $\mathrm{mm} \mathrm{NaCl}$, and $1 \mathrm{~mm}$ EDTA, supplemented with protease inhibitors (Sigma): $1 \mathrm{~mm} \mathrm{NaF}, 1 \mathrm{~mm} \mathrm{Na}_{3} \mathrm{VO}_{4}, 1 \mathrm{~mm}$ PMSF, leupeptin $(2 \mu \mathrm{g} / \mathrm{ml})$, aprotinin $(2 \mu \mathrm{g} / \mathrm{ml})$, and pepstatin A $(1 \mu \mathrm{g} / \mathrm{ml})]$ using a glass homogenizer. Protein concentrations were determined using BCA assay (Pierce). Equivalent amounts of proteins from wild-type and R6/1 transgenic mice were analyzed by SDS-PAGE and immunoblotting using antibodies as follows: CSP- $\alpha$ (R807), 1:1000; synaptobrevin 2/VAMP2 (Cl 69.1, Synaptic Systems), 1:1000; SNAP-25 (I733), 1:50; syntaxin 1A (I378, gift from Dr. Thomas Südhof (McMahon and Südhof, 1995), 1:50; dynamins 1-3 (Pan-Dyn, 115 002, Synaptic Systems), 1:500; and $\alpha$-actin (A 2066, Sigma), 1:1000, as loading control. Peroxidase-labeled secondary antibodies (Sigma and Biomedal) [anti-mouse (1:10,000) and anti-rabbit (1:5000)] were revealed using ECL Plus (GE Healthcare). Luminescence was acquired with a ChemiDoc XRS System (Bio-Rad). 

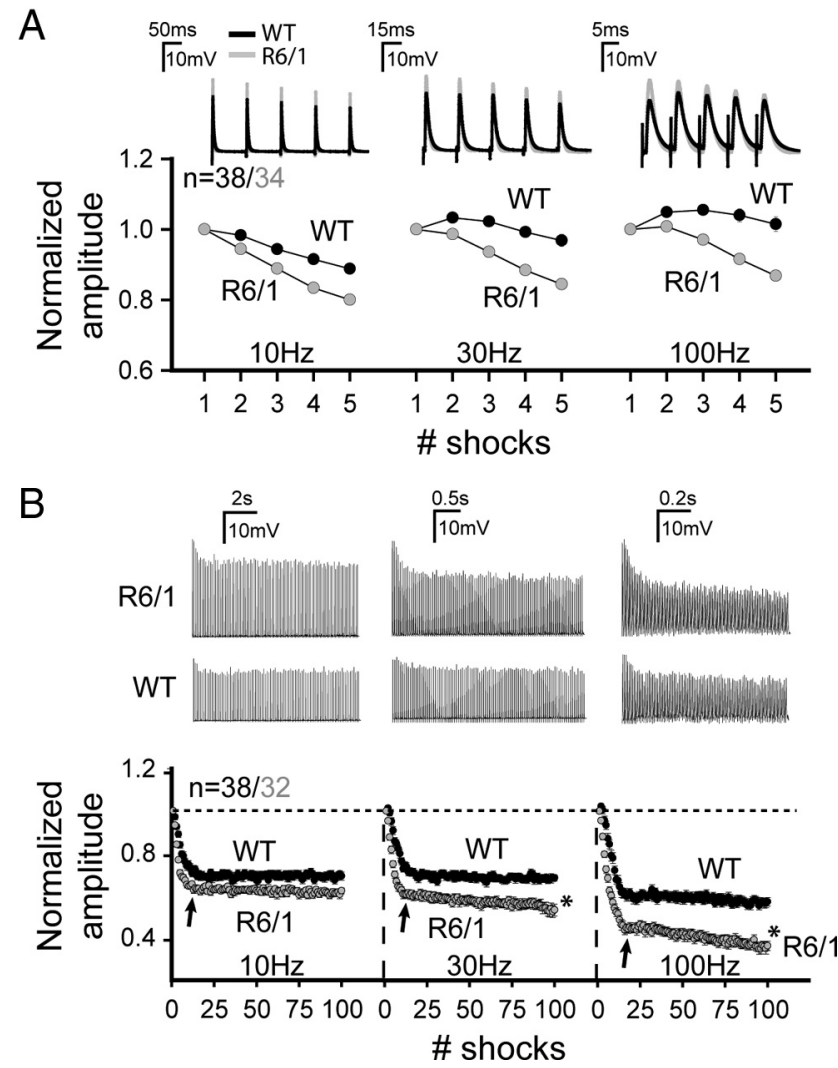

Figure 2. Strong synaptic depression at the R6/1 NMJ. $A$, Exemplary recordings of EPPs in response to electrical nerve stimulation at three different frequencies: 10,30 , and $100 \mathrm{~Hz}$ (top). Averaged responses (bottom) showed enhanced depression at R6/1 synapses compared with WT, a typical feature of synapses with high release probability. $\boldsymbol{B}$, Synapses were challenged with longer trains ( 100 action potentials, AP) at 10,30 , and $100 \mathrm{~Hz}$ to induce depletion of the readily releasable pool (RRP). Mutant synapses showed faster and deeper pool depletion, as expected for high release probability.

\section{Results}

Increased EPP amplitude and quantal content at the NMJ of R6/1 mice

We used the levator auris longus nerve-muscle preparation (Angaut-Petit et al., 1987) to characterize synaptic transmission at the NMJ of 5- to 6-month-old R6/1 transgenic mice. We found no significant changes in resting membrane potential $(-61.5 \pm$ $1.6 \mathrm{mV}, n=26$ in WT vs $-57.4 \pm 1.8 \mathrm{mV}, n=26$ in $\mathrm{R} 6 / 1, p=$ 0.11 , Student's $t$ test). First, we recorded MEPPs to examine spontaneous release (Fig. $1 A$ ) and found similar MEPP amplitudes in R6/1 and WT mice (Fig. $1 B, C)(0.73 \pm 0.08 \mathrm{mV}, n=26$ for WT; $0.73 \pm 0.04 \mathrm{mV}, n=26$ for R6/1), indicating no alterations in the acetylcholine content of single vesicles. MEPP frequency was slightly higher in the R6/1 mice but not significantly different from MEPP frequency in controls $(1.84 \pm 0.23 \mathrm{~Hz}$ in R6/1, $n=45$ vs $1.46 \pm 0.15 \mathrm{~Hz}$ in WT, $n=47$ ) (Fig. $1 D$ ). In contrast, EPPs, evoked by nerve stimulation using a suction electrode, turned out to have a dramatically higher amplitude in R6/1 junctions than EPPs recorded from control junctions (Fig. $1 F$ ) $(37.5 \pm 2.4 \mathrm{mV}, n=26$ for WT; $58.7 \pm 4.2 \mathrm{mV}, n=26$ for R6/1, $p<0.001$, Student's $t$ test). Quantal content estimation indicated that control nerve terminals released, on average, 50 vesicles per action potential, while terminals from $\mathrm{R} 6 / 1$ mice released $60 \%$ more vesicles: 80 vesicles per action potential (Fig. $1 F$ ). Thus, the augmented evoked release could be due to an increased readily releasable synaptic vesicle pool, to a higher release probability of every synaptic vesicle, or to both.
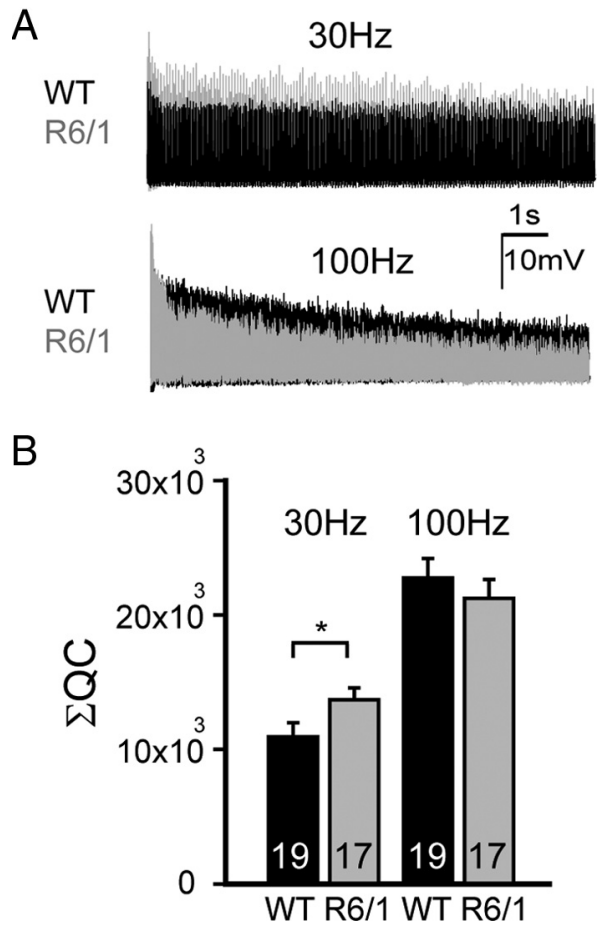

Figure 3. Enhanced release from a recycling synaptic vesicle pool with normal size. $A$, EPPs evoked by $10 \mathrm{~s}$ duration stimulation trains at $30 \mathrm{~Hz}$ (top) and $100 \mathrm{~Hz}$ (bottom) from WT (black) and $\mathrm{R} 6 / 1$ (gray) synapses. $\boldsymbol{B}$, The total number of quanta released during a train was obtained as the summation of every quanta released by each action potential. Total quanta released at $30 \mathrm{~Hz}$ was higher in mutant ( $24.9 \%$ increase) than in WT synapses $\left({ }^{*} p=0.035\right.$, Mann-Whitney test). However, no changes were observed at a stimulation frequency high enough $(100 \mathrm{~Hz})$ to release completely the recycling pool. The exemplary traces shown in $\boldsymbol{A}$ obtained in the same fibers yielded a cumulative QC at $30 \mathrm{~Hz}$ of 8978 quanta (WT) and 13,678 quanta (R6/1); at $100 \mathrm{~Hz}$, the values were 20,320 (WT) and 19,011 (R6/1) quanta.

\section{Strong short-term depression and normal recycling vesicle pool at $\mathrm{R} 6 / 1$ synapses}

Synapses with high release probability typically display high synaptic depression: the amplitude of postsynaptic responses becomes progressively lower during stimulation with highfrequency action potential trains. In contrast, synapses with lower release probability exhibit lower synaptic depression or even facilitation (Zucker and Regehr, 2002). Control synapses stimulated with trains of 5 action potentials at different frequencies responded with either a moderate synaptic depression (at 10 $\mathrm{Hz}$ stimulation frequency) or with synaptic facilitation followed by a weak depression (at 30 and $100 \mathrm{~Hz}$ stimulation frequencies) (Fig. 2A). In contrast, R6/1 synapses exhibited synaptic depression at every stimulation frequency that was significantly stronger than synaptic depression in control synapses (Fig. 2A). This observation is consistent with the notion that release probability is increased at R6/1 synapses. To test whether the underlying cause of such an increase was the existence of bigger synaptic vesicle pools, we challenged synapses with longer stimulation trains. Under those conditions, the efficient recycling and refilling of vesicle pools is required to sustain synaptic transmission during the train (Delgado et al., 2000; Rizzoli and Betz, 2005). EPP amplitude in control synapses dropped quickly at the beginning of the train to reach the steady-state amplitude maintained until the train ended (Fig. 2 B). The steady-state amplitude was lower at $100 \mathrm{~Hz}$, the highest frequency tested. R6/1 synapses responded similarly to wild-type synapses; however, the initial depression was deeper and the steady-state amplitudes reached lower values than at 
A
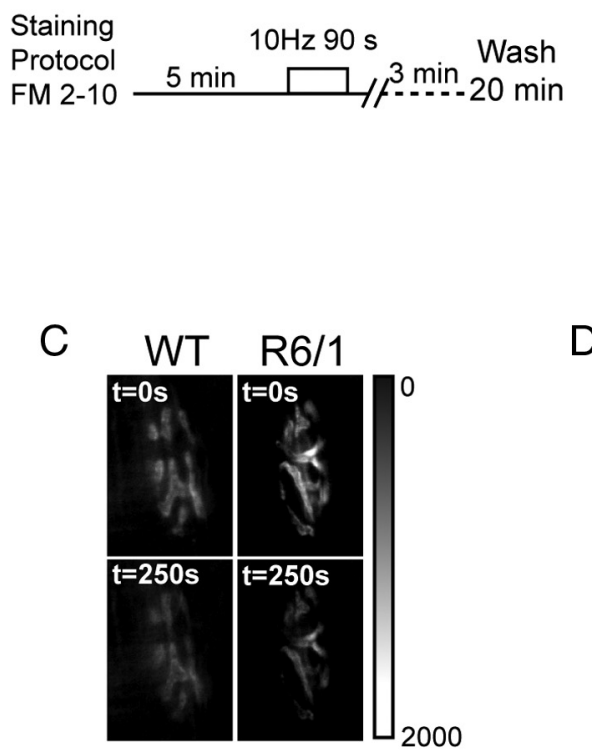

$E$

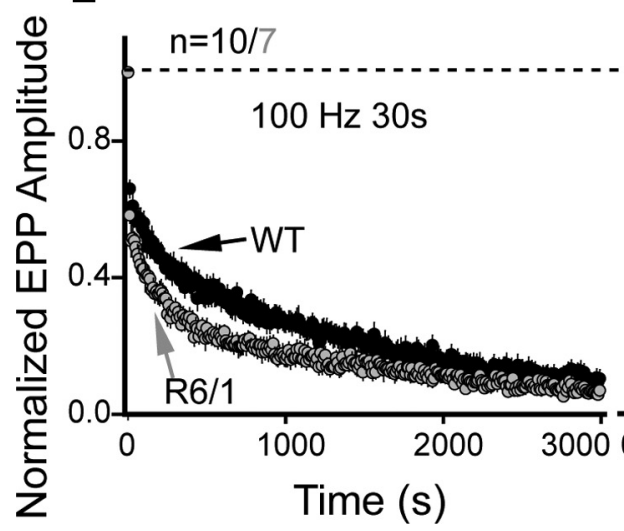

Figure 4. Imaging of enhanced release with FM2-10 styryl dye. $A$, NMJs were first loaded with FM2-10 by stimulating 90 s at 10 $\mathrm{Hz}$ and then challenged (separately) with different stimulation trains. $\boldsymbol{B}$, In response to $120 \mathrm{~s}$ at $50 \mathrm{~Hz}$ stimulation train, both WT and R6/1 synapses lost the FM2-10 dye almost completely. No obvious changes in destaining kinetics were observed between WT and R6/1. C, D, Upon milder stimulation with $40 \mathrm{~s}$ at $10 \mathrm{~Hz}$ trains, mutant synapses destained more and faster than WT synapses, as expected for higher release probability at R6/1 synapses. $\boldsymbol{E}$, Recycling pool recovery from depletion (induced by a $30 \mathrm{~s}$ at $100 \mathrm{~Hz}$ train) was assessed by measuring EPP amplitude evoked by sequential stimulation at $2 \mathrm{~Hz}$. Although the depression is stronger for R6/1 synapses (for clarity, only 1 point of 10 is drawn), the recovery was very similar in WT and mutant synapses. Thus, no obvious abnormalities occurred in the refilling of the recycling pool at R6/1 synapses.

wild-type synapses (Fig. 2B, arrows). Furthermore, for high stimulation frequencies ( 30 and $100 \mathrm{~Hz}$ ), EPP amplitudes in R6/1 synapses failed to hold a constant steady-state level and the amplitude progressively ran down until the end of the train (Fig. $2 B$, asterisks). In addition, out of this set of data, we generated cumulative plots of the quantal content released during the stimulation train and attempted to extrapolate the size of the readily releasable synaptic vesicle pool as previously described in central synapses (Schneggenburger et al., 1999; Moulder and Mennerick, 2005). However, that approach was not valid in our experiments, because recovery from depression was not negligible for the initial time interval before reaching the steady-state phase of depression (data not shown). In any case, our data did not support the existence of bigger recycling synaptic vesicle pools or increased rate of pool refilling from a putative reserve pool
(Poskanzer and Davis, 2004; Rizzoli and Betz, 2005). Instead, our data, again, exposed a greater avidness of $\mathrm{R} 6 / 1$ synapses for evoked release that was high enough to compromise recycling mechanisms that could not cope with the augmented synaptic vesicle exocytosis. However, those observations are rather indirect and did not rule out that, concomitant with the enhanced release, R6/1 synapses carried a thinner recycling pool. To get insight into that possibility, we computed the total quantal content released upon nerve stimulation during $10 \mathrm{~s}$ at 30 and $100 \mathrm{~Hz}$. Stimulating at $30 \mathrm{~Hz}$, the total number of vesicles released was significantly higher in R6/1 synapses (Fig. 3A,B). However, for stronger stimulation rate at $100 \mathrm{~Hz}$, although control and R6/1 junctions released more vesicles than at $30 \mathrm{~Hz}$, the R6/1 and control synapses behaved alike (Fig. 3A,B). Probably, we detected a higher number of released vesicles from R6/1 terminals at $30 \mathrm{~Hz}$, and not at 100 $\mathrm{Hz}$, because at a lower frequencies vesicle recycling is likely not a limiting step for exocytosis. In addition, we also used those recordings to measure the MEPP frequency at the end of every train and found that, under those conditions, the frequency of MEPPs was increased in R6/1 junctions (after $30 \mathrm{~Hz}$ trains: $4.54 \pm 0.65$ $\mathrm{Hz}$ in $\mathrm{R} 6 / 1, n=35$ vs $2.98 \pm 0.93 \mathrm{~Hz}$ in WT, $n=37, p=0.05$, Student's $t$ test; after $100 \mathrm{~Hz}$ trains: $10.09 \pm 1.20$ in R6/1, $n=35$ vs $6.86 \pm 0.79 \mathrm{~Hz}$ in WT, $n=37$, $p=0.02$, Student's $t$ test). Furthermore, we set up conditions for imaging release with the styryl dyes FM2-10 (Ribchester et al., 1994; Perissinotti et al., 2008). Dye loading into motor neuron terminals incubated with FM2-10 was performed by stimulating synaptic vesicle exocytosis and endocytosis with a long train of action potentials ( $90 \mathrm{~s}$ at $10 \mathrm{~Hz}$ ) (Fig. $4 \mathrm{~A}$ ), followed by extensive wash to remove noninternalized dye. Subsequent strong nerve stimulation $(120 \mathrm{~s}$ at $50 \mathrm{~Hz})$ resulted in almost complete destaining of the terminals, as expected for the emptying of the recycling pool of synaptic vesicles (Fig. $4 B$ ). Destaining took place with the same kinetics and amplitude in wild-type and R6/1 junctions (Fig. 4B). However, stimulation with shorter trains at lower frequencies ( $40 \mathrm{~s}$ at $10 \mathrm{~Hz}$ ), to release only a fraction of the recycling vesicle pool, led to faster and stronger destaining of R6/1 terminals than of controls (Fig. 4C,D). Notably, those observations, consistently with the electrophysiological measurements, confirmed that vesicle release from R6/1 terminals was strikingly heightened. Moreover, those results pointed to similar size of the recycling pool of synaptic vesicles in wild-type and R6/1 terminals. In addition, consistent with normal recycling mechanisms, we found no changes in the recovery of synaptic responses after induction of strong synaptic depression (30 s at $10 \mathrm{~Hz})($ Fig. $4 E)$. 
A

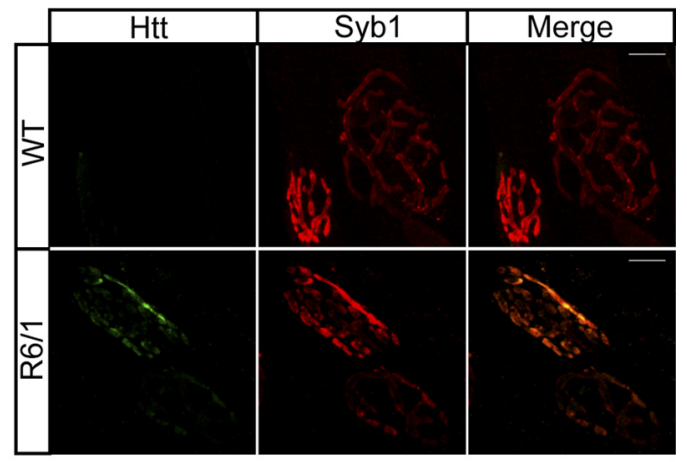

B

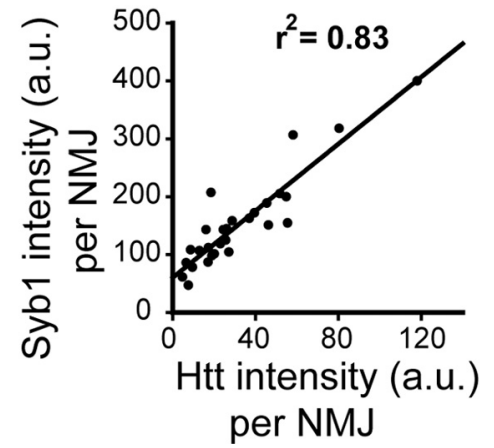

Figure 5. Direct correlation between the expression of mutant-htt and synaptobrevin 1/VAMP1 in R6/1 synapses. $\boldsymbol{A}$, Htt immunoreactivity was not detected at the NMJ in WT mice. In contrast, the same antibody labeled R6/1 junctions, as expected for expanded-htt expression at the synapse. Htt labeling was unevenly distributed among different junctions, suggesting a variegated expression pattern. $\boldsymbol{B}$, Linear relationship between htt and synaptobrevin 1 expression. Scale bars, $10 \mu \mathrm{m}$.

Synaptic overexpression of synaptobrevin 1,2/VAMP 1,2, CSP- $\alpha$, and SNAP-25 at the R6/1 motor terminals

Since the synaptic phenotype that we have found might be mediated by a direct effect of htt on the molecular machinery of synaptic vesicle exocytosis, we searched for htt expression at the NMJ. Using specific fluorescently labeled antibodies against htt, we could not detect any expression at the wild-type junctions. In contrast, at the R6/1 junctions, htt expression was high enough to be clearly detected with immunohistochemical techniques in many junctions (Fig. 5A). In any case, htt expression was not uniformly distributed among all the junctions and, instead, exhibited a variegated pattern. As a control marker for immunohistochemistry, we used antibodies against the synaptic vesicle protein synaptobrevin 1/VAMP1 (Fig. 5A). The synaptic surface labeled with synaptobrevin 1/VAMP1 antibodies were similar in control and R6/1 mice (163.5 $\pm 11.5 \mu \mathrm{m}^{2}, n=64$ for controls and $169.8 \pm 7.2 \mu \mathrm{m}^{2}, n=76$ for R6/1), suggesting that the terminals were not degenerated. Unexpectedly, however, the signal intensity for synaptobrevin 1/VAMP1 was apparently stronger in the mutants than in the controls. Indeed, a closer examination of junctions doubly labeled with antibodies against htt and synaptobrevin 1 revealed a linear relationship between the intensity of the two markers (Fig. 5B). Next, we performed double labeling using antibodies against synaptobrevin 1/VAMP1 and against another synaptic vesicle protein, synaptic vesicle protein 2 (SV2) (Fig. 6A). Interestingly, quantification of both signal intensities demonstrated a significant increase in the intensity of synaptobrevin 1/VAMP1 staining normalized to SV2 staining (Fig. 6D). Next, we extended our study to other synaptic vesicle proteins and found normal levels of other synaptic vesicle markers such as synaptophysin 1 (Fig. 6B) and synaptotagmin 1,2 , but not for CSP- $\alpha$, which was unexpectedly increased. In addition, we checked the levels of the SNARE proteins SNAP-25 and syntaxin 1 A,B. Surprisingly, we observed a significant increase in the SNAP-25 staining at the R6/1 junctions (Fig. 6C,D). In contrast, we could not detect changes of fluorescence intensity for syntaxin $1 \mathrm{~A}, \mathrm{~B}$; however, since the signal obtained with antisyntaxin $1 \mathrm{~A}, \mathrm{~B}$ antibodies was rather weak and with higher background than for the other markers (data not shown), we decided to complement our study with immunoblots. In contrast to the Western blot analysis of synaptic protein levels in brain, the analysis of synaptic proteins from the neuromuscular junction at the tiny levator auris longus muscle is challenging because the amount of synaptic proteins is very low. We have nevertheless set up conditions to detect proteins by Western blot in neuromuscular preparations from R6/1 and control mice by pooling the protein extracts from several mice. In agreement with the immunofluorescence experiments, we have found stronger signals for SNAP-25 and CSP- $\alpha$ in protein extracts from R6/1 mice. Other proteins, such as syntaxin 1A, dynamins (1-3), and actin, did not show any obvious change. We also tested the antibody against synaptobrevin 1 that we successfully used for immunofluorescence staining, but unfortunately we did not detect any signal, indicating that the antibody does not work for our immunoblots. Then, we decided to check synaptobrevin 2/VAMP2 levels in Western blots and, interestingly, found that the levels of synaptobrevin 2/VAMP2 were increased in the $\mathrm{R} 6 / 1$ protein extracts. In summary, among all the proteins studied, we did not detect any decrease in the levels of any of them; however, synaptobrevin 1,2/VAMP 1,2, CSP- $\alpha$, and SNAP-25 turned out to be increased at the junctions of R6/1 mice.

\section{Discussion}

Increased quantal content and strong synaptic depression We have found a gain-of-function phenotype in neurotransmitter release at the motor nerve terminals of R6/1 transgenic mice, a mouse model of polyglutamine disease. In the context of previous studies of synaptic function in $\mathrm{HD}$, our findings are novel because they demonstrate for the first time that expanded htt increases synaptic release at vertebrate motor nerve terminals. We have shown that, at the NMJ of R6/1 mice, there are no differences in MEPP amplitude, but the amplitude of evoked release is $60 \%$ higher than in control mice (Fig. 1). To minimize errors in the quantal content estimation, we have corrected the effect of nonlinear summation on EPP amplitude as previously described (McLachlan and Martin, 1981) using a correction factor $(f=0.8)$ (see Materials and Methods). The introduction of that factor improves overcorrection introduced by older methods (Martin, 1955), and it has been used in different mouse neuromuscular preparations (Plomp et al., 1992; Wood and Slater, 1997; Bullens et al., 2002; Bewick et al., 2004; Chen et al., 2010). Current-clamp measurements of quantal content could be relatively inaccurate if compared with absolute quantal content values obtained with voltage-clamp recordings as previously described in other preparations (Wood and Slater, 1997). However, we do not have any reason to suspect that the significant increase in quantal content that we have found in R6/1 synapses would be different if we measured synaptic transmission with voltage clamp. Furthermore, as expected for synapses with high release probability, R6/1 synapses undergo strong synaptic depression (Fig. 2). We cannot rule out that the number of release sites, regarded as the number of morphologically defined active zones, is increased. However, that change alone would not be enough to 

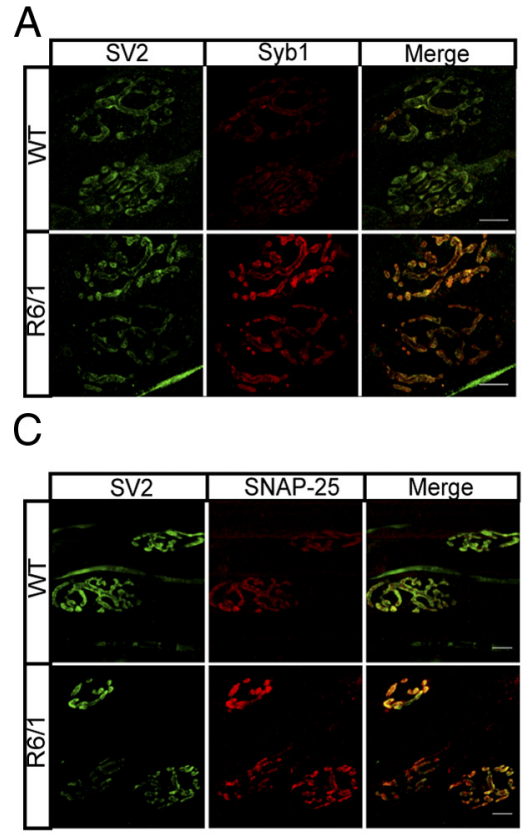

D
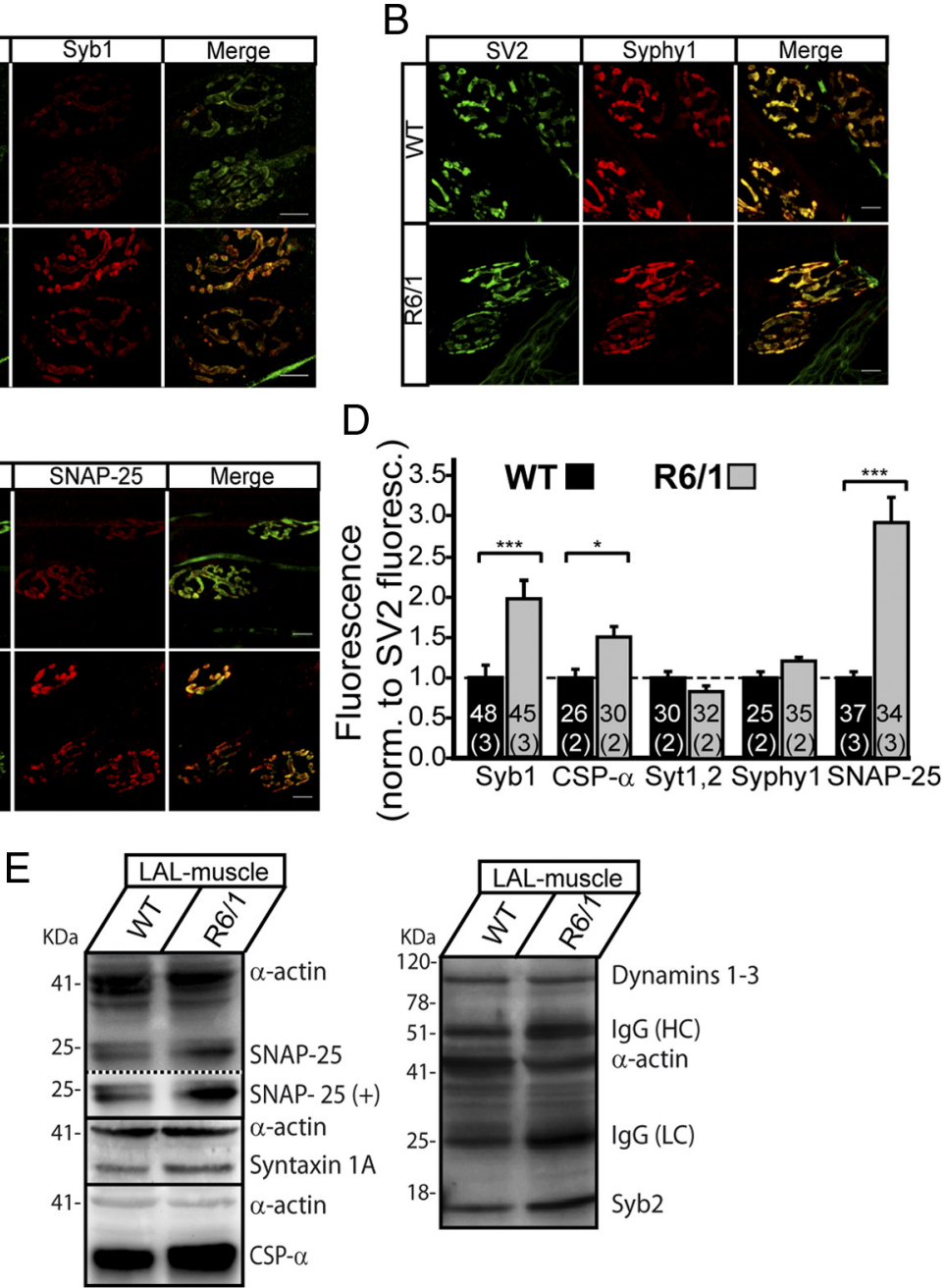

Figure 6. Synaptic overexpression of synaptobrevin 1,2/NAMP 1,2, CSP- $\alpha$, and SNAP-25 at the R6/1 motor terminals. $\boldsymbol{A}, \boldsymbol{B}$, Increase of synaptobrevin 1/VAMP1 (Syb1) but not of synaptophysin 1 (Syphy1) in mutant synapses, compared with SV2. C, Increased levels of SNAP-25 at R6/1 synapses. D, Quantitation of fluorescence intensity for several synaptic proteins normalized to SV2 fluorescence in WT and R6/1 mutant junctions. (Significant differences: ${ }^{*} p=0.026$ or ${ }^{* * *} p<0.001$, Student's $t$ test). $\boldsymbol{E}_{\text {, }}$ Immunoblots from protein extracts of LAL from WT and R6/1 mice reveals increased levels of SNAP-25, CSP- $\alpha$, and Syb2. No obvious changes are detected in dynamins $1-3$, syntaxin $1 A$, or $\alpha$-actin. The secondary antibodies against mouse lg $G$ reacts with

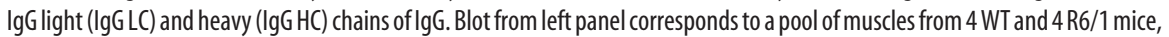
and blot from right panel corresponds to a pool from 2 WT and 2 R6/1 mice. Scale bars, $10 \mu \mathrm{m}$. The sign (+) means longer exposure.

explain the phenotype. An increase in the number of release sites without increasing the probability that vesicle fusion occurs at a given active zone should not lead to higher synaptic depression. Consistent with an unchanged number of release sites, we do not see changes in the synaptic surface area between R6/1 and controls; however, in future studies, electron microscopy analysis will be useful to get deeper insight into that issue.

\section{Normal recycling vesicle pool at R6/1 synapses}

In addition, we have used electrophysiology and imaging to observe that the dynamics and vesicle pool size are similar in R6/1 and control synapses. (1) The total number of quanta released, at the end of a train, is higher at R6/1 synapses at $30 \mathrm{~Hz}$ but equal to controls at $100 \mathrm{~Hz}$ (Fig. 3). Likely, at the higher frequency, the limiting step for release is not exocytosis but the similar recycling capacity (Denker and Rizzoli, 2010) in mutant and control synapses. (2) Destaining of styryl dyes from R6/1 terminals, induced by short stimulation trains, is higher and faster than in controls.
In contrast, destaining due to the release of the total recycling pool, induced by stronger stimulation trains (Perissinotti et al., 2008), was identical in R6/1 and control terminals (Fig. 4). (3) The time course of the recovery of EPP amplitudes after synaptic depression was the same for both genotypes, consistent with the notion that vesicle recycling at R6/1 terminals is not altered (Fig. 4). In summary, the gain of function that we have observed does not come from a bigger or faster refilling of the recycling synaptic vesicle pool.

\section{Release enhancement not due to neurodegeneration}

We have not found morphological signs of nerve terminal damage; therefore, the phenotype is likely not due to secondary neurodegenerative alterations. Indeed, it would be difficult to explain how synaptic run-down caused by neurodegeneration would translate into enhancement of release. R6/2 mice undergo fast and progressive NMJ degeneration characterized by early muscle atrophy and robust synaptic transmission, followed by neurotransmitter release impairment before death (Ribchester et al., 2004). In contrast to our observation in R6/1 mice, motor nerve terminals of R6/2 mice apparently do not have any increase in neurotransmitter release. Perhaps such a phenotype might be masked or counterbalanced by the stronger degeneration in R6/2 mice. Nevertheless, our findings at the NMJ of R6/1 mice are in agreement with the large size of evoked EPSCs and the decreased pairedpulse ratio at some stimulation frequencies described in cortical synapses from R6/2 mice (Cummings et al., 2009). In addition, our findings are consistent with a recent study on a novel Drosophila HD model expressing human full-length htt with a 128 polyQ expansion $\left(128 \mathrm{Qhtt}{ }^{\mathrm{FL}}\right.$ ) (Romero et al., 2008). Similar to our observations in R6/1 mice, motor neurons from larvae expressing $128 \mathrm{Qhtt}{ }^{\mathrm{FL}}$ exhibited normal spontaneous release and a significant increase in the amplitude of evoked excitatory junction potentials. Interestingly, adult flies expressing $128 \mathrm{Qhtt}{ }^{\mathrm{FL}}$ developed degeneration of motor neurons that was prevented upon genetic manipulations that reduced exocytosis (Romero et al., 2008). Strikingly, the increase in neurotransmitter release that we observe in R6/1 does not require the polyQ expansion within the full-length htt, as occurs at the fly $128 \mathrm{Qhtt}{ }^{\mathrm{FL}}$ model. That implies that the mere polyQ expansion at the $\mathrm{N}$-terminal portion of $\mathrm{htt}$, and not necessarily the full-length, might be sufficient to induce the synaptic phenotype. This notion could be relevant to investigate synaptic phenotypes in other models of polyQ diseases. Future experiments should test whether genetic or pharmacological synaptic manipulation in R6/1 mice at early phases could indeed prevent neuronal degeneration. 


\section{Alterations in $\mathrm{Ca}^{2+}$ release from intracellular stores?}

Tang et al. $(2003,2005)$ have demonstrated that expanded htt binds and facilitates activity of type 1 inositol 1,4,5-triphosphate receptor (InsP3R1), indicating that abnormally increased neuronal $\mathrm{Ca}^{2+}$ levels may be important in HD pathogenesis. We cannot rule out the possibility that mutant-htt might enhance neurotransmitter release by promoting $\mathrm{Ca}^{2+}$ release from intracellular stores at the motor nerve terminals of R6/1 mice. If that were the case, we should detect a $\mathrm{Ca}^{2+}$-dependent elevation in the MEPP frequency in resting conditions (Llano et al., 2000; Xu et al., 2009); however, we detected that MEPP frequency was slightly, but not significantly, higher at the R6/1 terminals (Fig. $1 D)$. In contrast, Romero et al. (2008) reported elevated resting synaptic $\mathrm{Ca}^{2+}$ levels at the $128 \mathrm{Qhtt}{ }^{\mathrm{FL}}$ strain with no changes in the frequency of spontaneous release (for review, see Rozas et al., 2010). We nevertheless recorded a higher MEPP frequency in R6/1 junctions after high-frequency stimulation trains, but we cannot distinguish whether such an increase is due to (1) abnormally high cytosolic $\left[\mathrm{Ca}^{2+}\right],(2)$ an effect downstream of a $\mathrm{Ca}^{2+}$. dependent step of exocytosis activation, or (3) both. Future experiments will have to address that question.

\section{Increased levels of synaptic SNAREs and CSP- $\alpha$}

Interestingly, since $128 \mathrm{Qhtt}{ }^{\mathrm{FL}}$ accumulated in the cytoplasm and never in the nucleus, Romero et al. (2008) considered that potential transcriptional alterations were not involved in the synaptic phenotype in the fly. Since we have detected htt immunoreactivity at the NMJ of R6/1 mice, we favor the hypothesis of a direct action of htt likely mediated by protein-protein interactions at the synapse. Our most surprising molecular observation is the increased levels of specific synaptic proteins, the SNAREs synaptobrevin 1,2/VAMP 1,2 and SNAP-25, and the synaptic vesicle protein CSP- $\alpha$ (Fig. 6). Such a specific protein change cannot be explained by a general or uniform decrease, which could be, for example, expected to occur as a consequence of severe synaptic damage. Instead, expanded htt, by unknown mechanisms, leads to selective alterations of, at least, a few synaptic proteins. A previous study has shown a selective decrease of the synaptic vesicle protein vesicular acetylcholine transporter (VAChT) concomitant with a normal level of synaptophysin at the NMJ of R6/1 mice (Smith et al., 2006). We do not know the mechanism by which expanded htt might selectively increase the level of synaptic proteins. However, we have recently shown that the expandedhtt expression induces a transient impairment of the ubiquitin/ proteasome system (UPS) that is rescued upon sequestration of expanded htt in protein aggregates (Ortega et al., 2010). On the other hand, Wang et al. (2008) have shown that synaptic proteins (SNAP-25 and PSD-95) fused to a fluorescent reporter of UPS activity do not undergo degradation in R6/2 synapses. Therefore, the general transient impairment of the UPS could become a rather long-lasting impairment at the synaptic terminals, perhaps because the microtubule-dependent sequestration of expanded htt (Kopito, 2000; Muchowski et al., 2002) is less efficient at the synaptic compartments. In addition, by unknown molecular mechanisms, pharmacological inhibition of the UPS increases neurotransmitter release in hippocampal cultures (Willeumier et al., 2006; Rinetti and Schweizer, 2010). Although we do not have yet enough elements to build up a mechanistic molecular model to explain the enhanced release in R6/1 synapses, it is likely that the upregulation of two SNARE proteins (synaptobrevins and SNAP-25) might be part of the molecular changes contributing to the gain of function phenotype in neurotransmitter release. On the other hand, $\operatorname{CSP}-\alpha$, which has been shown to interact with mutant-htt (Miller et al., 2003), is required to maintain SNAP-25 levels (Chandra et al., 2005). Both proteins, CSP- $\alpha$ and SNAP-25, become reduced in Drosophila mutants lacking the palmitoyl transferase huntingtin-interacting protein 14 (Ohyama et al., 2007; Stowers and Isacoff, 2007). Therefore, in future studies, it would be interesting to explore the functional significance of a parallel synaptic increase of CSP- $\alpha$ and SNAP-25 in mouse models of polyglutamine diseases.

\section{References}

Angaut-Petit D, Molgo J, Connold AL, Faille L (1987) The levator auris longus muscle of the mouse: a convenient preparation for studies of short- and long-term presynaptic effects of drugs or toxins. Neurosci Lett 82:83-88

Bewick GS, Reid B, Jawaid S, Hatcher T, Shanley L (2004) Postnatal emergence of mature release properties in terminals of rat fast- and slow-twitch muscles. Eur J Neurosci 19:2967-2976.

Bullens RW, O’Hanlon GM, Wagner E, Molenaar PC, Furukawa K, Furukawa K, Plomp JJ, Willison HJ (2002) Complex gangliosides at the neuromuscular junction are membrane receptors for autoantibodies and botulinum neurotoxin but redundant for normal synaptic function. J Neurosci 22: $6876-6884$.

Cepeda C, Hurst RS, Calvert CR, Hernández-Echeagaray E, Nguyen OK, Jocoy E, Christian LJ, Ariano MA, Levine MS (2003) Transient and progressive electrophysiological alterations in the corticostriatal pathway in a mouse model of Huntington's disease. J Neurosci 23:961-969.

Cepeda C, Wu N, André VM, Cummings DM, Levine MS (2007) The corticostriatal pathway in Huntington's disease. Prog Neurobiol 81:253-271.

Chandra S, Gallardo G, Fernández-Chacón R, Schlüter OM, Südhof TC (2005) Alpha-synuclein cooperates with CSPalpha in preventing neurodegeneration. Cell 123:383-396.

Chen F, Sugiura Y, Myers KG, Liu Y, Lin W (2010) Ubiquitin carboxylterminal hydrolase $\mathrm{L} 1$ is required for maintaining the structure and function of the neuromuscular junction. Proc Natl Acad Sci U S A 107:16361641.

Cummings DM, André VM, Uzgil BO, Gee SM, Fisher YE, Cepeda C, Levine MS (2009) Alterations in cortical excitation and inhibition in genetic mouse models of Huntington's disease. J Neurosci 29:10371-10386.

Delgado R, Maureira C, Oliva C, Kidokoro Y, Labarca P (2000) Size of vesicle pools, rates of mobilization, and recycling at neuromuscular synapses of a Drosophila mutant, shibire. Neuron 28:941-953.

Denker A, Rizzoli SO (2010) Synaptic vesicle pools: an update. Front Syn Neurosci 2:135.

Fatt P, Katz B (1952) Spontaneous subthreshold activity at motor nerve endings. J Physiol 117:109-128.

Fernández-Chacón R, Wölfel M, Nishimune H, Tabares L, Schmitz F, Castellano-Muñoz M, Rosenmund C, Montesinos ML, Sanes JR, Schneggenburger R, Südhof TC (2004) The synaptic vesicle protein CSP alpha prevents presynaptic degeneration. Neuron 42:237-251.

Kopito RR (2000) Aggresomes, inclusion bodies and protein aggregation. Trends Cell Biol 10:524-530.

Llano I, González J, Caputo C, Lai FA, Blayney LM, Tan YP, Marty A (2000) Presynaptic calcium stores underlie large-amplitude miniature IPSCs and spontaneous calcium transients. Nat Neurosci 3:1256-1265.

Magleby KL, Stevens CF (1972a) The effect of voltage on the time course of end-plate currents. J Physiol 223:151-171.

Magleby KL, Stevens CF (1972b) A quantitative description of end-plate currents. J Physiol 223:173-197.

Mangiarini L, Sathasivam K, Seller M, Cozens B, Harper A, Hetherington C, Lawton M, Trottier Y, Lehrach H, Davies SW, Bates GP (1996) Exon 1 of the HD gene with an expanded CAG repeat is sufficient to cause a progressive neurological phenotype in transgenic mice. Cell 87:493-506.

Martin AR (1955) A further study of the statistical composition on the endplate potential. J Physiol 130:114-122.

Martin AR (1976) The effect of membrane capacitance on non-linear summation of synaptic potentials. J Theor Biol 59:179-187.

McLachlan EM, Martin AR (1981) Non-linear summation of end-plate potentials in the frog and mouse. J Physiol 311:307-324.

McMahon HT, Südhof TC (1995) Synaptic core complex of synaptobrevin, syntaxin, and SNAP25 forms high affinity alpha-SNAP binding site. J Biol Chem 270:2213-2217. 
Miller LC, Swayne LA, Chen L, Feng ZP, Wacker JL, Muchowski PJ, Zamponi GW, Braun JE (2003) Cysteine string protein (CSP) inhibition of N-type calcium channels is blocked by mutant huntingtin. J Biol Chem 278:53072-53081.

Moulder KL, Mennerick S (2005) Reluctant vesicles contribute to the total readily releasable pool in glutamatergic hippocampal neurons. J Neurosci 25:3842-3850.

Muchowski PJ, Ning K, D'Souza-Schorey C, Fields S (2002) Requirement of an intact microtubule cytoskeleton for aggregation and inclusion body formation by a mutant huntingtin fragment. Proc Natl Acad Sci U S A 99:727-732.

Ohyama T, Verstreken P, Ly CV, Rosenmund T, Rajan A, Tien AC, Haueter C, Schulze KL, Bellen HJ (2007) Huntingtin-interacting protein 14, a palmitoyl transferase required for exocytosis and targeting of CSP to synaptic vesicles. J Cell Biol 179:1481-1496.

Orr HT, Zoghbi HY (2007) Trinucleotide repeat disorders. Annu Rev Neurosci 30:575-621.

Ortega Z, Díaz-Hernández M, Maynard CJ, Hernández F, Dantuma NP, Lucas JJ (2010) Acute polyglutamine expression in inducible mouse model unravels ubiquitin/proteasome system impairment and permanent recovery attributable to aggregate formation. J Neurosci 30: 3675-3688.

Perissinotti PP, Giugovaz Tropper B, Uchitel OD (2008) L-type calcium channels are involved in fast endocytosis at the mouse neuromuscular junction. Eur J Neurosci 27:1333-1344.

Plomp JJ, van Kempen GT, Molenaar PC (1992) Adaptation of quantal content to decreased postsynaptic sensitivity at single endplates in alphabungarotoxin-treated rats. J Physiol 458:487-499.

Poskanzer KE, Davis GW (2004) Mobilization and fusion of a non-recycling pool of synaptic vesicles under conditions of endocytic blockade. Neuropharmacology 47:714-723.

Ribchester RR, Mao F, Betz WJ (1994) Optical measurements of activitydependent membrane recycling in motor nerve terminals of mammalian skeletal muscle. Proc Biol Sci 255:61-66.

Ribchester RR, Thomson D, Wood NI, Hinks T, Gillingwater TH, Wishart TM, Court FA, Morton AJ (2004) Progressive abnormalities in skeletal muscle and neuromuscular junctions of transgenic mice expressing the Huntington's disease mutation. Eur J Neurosci 20:3092-3114.

Rinetti GV, Schweizer FE (2010) Ubiquitination acutely regulates presynaptic neurotransmitter release in mammalian neurons. J Neurosci 30:3157-3166.

Rizzoli SO, Betz WJ (2005) Synaptic vesicle pools. Nat Rev Neurosci 6:57-69.

Romero E, Cha GH, Verstreken P, Ly CV, Hughes RE, Bellen HJ, Botas J (2008) Suppression of neurodegeneration and increased neurotransmission caused by expanded full-length huntingtin accumulating in the cytoplasm. Neuron 57:27-40.

Rozas JL, Gómez-Sánchez L, Tomás-Zapico C, Lucas JJ, Fernández-Chacón R (2010) Presynaptic dysfunction in Huntington's disease. Biochem Soc Trans 38:488-492.
Ruiz-Montasell B, Aguado F, Majó G, Chapman ER, Canals JM, Marsal J, Blasi J (1996) Differential distribution of syntaxin isoforms 1A and 1B in the rat central nervous system. Eur J Neurosci 8:2544-2552.

Schneggenburger R, Meyer AC, Neher E (1999) Released fraction and total size of a pool of immediately available transmitter quanta at a calyx synapse. Neuron 23:399-409.

Smith R, Brundin P, Li JY (2005) Synaptic dysfunction in Huntington's disease: a new perspective. Cell Mol Life Sci 62:1901-1912.

Smith R, Chung H, Rundquist S, Maat-Schieman ML, Colgan L, Englund E, Liu YJ, Roos RA, Faull RL, Brundin P, LiJY (2006) Cholinergic neuronal defect without cell loss in Huntington's disease. Hum Mol Genet 15:3119-3131

Stowers RS, Isacoff EY (2007) Drosophila huntingtin-interacting protein 14 is a presynaptic protein required for photoreceptor synaptic transmission and expression of the palmitoylated proteins synaptosome-associated protein 25 and cysteine string protein. J Neurosci 27:12874-12883.

Tang TS, Tu H, Chan EY, Maximov A, Wang Z, Wellington CL, Hayden MR, Bezprozvanny I (2003) Huntingtin and huntingtin-associated protein 1 influence neuronal calcium signaling mediated by inositol- $(1,4,5)$ triphosphate receptor type 1. Neuron 39:227-239.

Tang TS, Slow E, Lupu V, Stavrovskaya IG, Sugimori M, Llinás R, Kristal BS, Hayden MR, Bezprozvanny I (2005) Disturbed Ca2 + signaling and apoptosis of medium spiny neurons in Huntington's disease. Proc Natl Acad Sci U S A 102:2602-2607.

The Huntington's Disease Collaborative Research Group (1993) A novel gene containing a trinucleotide repeat that is expanded and unstable on Huntington's disease chromosomes. Cell 72:971-983.

Ullrich B, Südhof TC (1994) Distribution of synaptic markers in the retina: implications for synaptic vesicle traffic in ribbon synapses. J Physiol Paris 88:249-257.

Wang J, Wang CE, Orr A, Tydlacka S, Li SH, Li XJ (2008) Impaired ubiquitin-proteasome system activity in the synapses of Huntington's disease mice. J Cell Biol 180:1177-1189.

Willeumier K, Pulst SM, Schweizer FE (2006) Proteasome inhibition triggers activity-dependent increase in the size of the recycling vesicle pool in cultured hippocampal neurons. J Neurosci 26:11333-11341.

Wood SJ, Slater CR (1997) The contribution of postsynaptic folds to the safety factor for neuromuscular transmission in rat fast- and slow-twitch muscles. J Physiol 500:165-176.

Xu J, Pang ZP, Shin OH, Südhof TC (2009) Synaptotagmin-1 functions as a $\mathrm{Ca} 2+$ sensor for spontaneous release. Nat Neurosci 12:759-766.

Zeron MM, Hansson O, Chen N, Wellington CL, Leavitt BR, Brundin P, Hayden MR, Raymond LA (2002) Increased sensitivity to N-methyl-Daspartate receptor-mediated excitotoxicity in a mouse model of Huntington's disease. Neuron 33:849-860.

Zucker RS, Regehr WG (2002) Short-term synaptic plasticity. Annu Rev Physiol 64:355-405. 